\title{
THE BOUNDARY INTEGRAL EQUATION METHOD IN PLANE ELASTICITY
}

\author{
CHRISTIAN CONSTANDA
}

(Communicated by Palle E. T. Jorgensen)

\begin{abstract}
The boundary integral equation method in terms of real variables is applied to solve the interior and exterior Dirichlet and Neumann problems of plane elasticity. In the exterior case, a special far-field pattern for the displacements is considered, without which the classical scheme fails to work. The connection between the results obtained by means of this technique and those of the direct method is indicated.
\end{abstract}

\section{INTRODUCTION}

Boundary value problems for the equations of plane elasticity have been extensively investigated via integral equations in the complex domain (see, for example, [1]). While the complex variable technique is very powerful and elegant, it has the drawback that its essential ingredients must be constructed in full for every individual situation, which often turns out to be an onerous task. By contrast, the real variable alternative does not suffer from this inconvenience, its generality allowing it to be used successfully for solving a large class of linear elliptic boundary value problems, with only modifications of detail from one case to another.

In spite of its practical relevance, plane elasticity has largely been neglected in the literature devoted to the real boundary integral equation method, where attention is mainly focused on the three-dimensional theory. This is clearly an injustice, since it is plane elasticity that proves to be mathematically the more challenging of the two, owing to the fact that its fundamental solutions do not decay to zero at infinity, as required in the general scheme.

An attempt at a systematic solution of the fundamental boundary value problems for the equations of plane strain in terms of real variables can be found in [2]. The far-field pattern considered there for the solution $u$ of the exterior problems is

$$
u=O(1), \quad \frac{\partial u}{\partial R}=O\left(R^{-2}\right) \quad \text { as } \quad R \rightarrow \infty .
$$

Under this assumption, the solution of the exterior Neumann problem is unique

Received by the editors April 18, 1994.

1991 Mathematics Subject Classification. Primary 35J55, 45E05, 73C35.

Key words and phrases. Boundary integral equation, plane elasticity. 
only up to a constant vector. Existence theorems, though, are not proved explicitly, being passed over with the mention that they are handled exactly as in the three-dimensional case. But the corresponding proofs for three-dimensional elasticity do not carry over automatically to the plane theory: in the latter, the single-layer potential $V$ is $O(\ln R)$ as $R \rightarrow \infty$ and needs additional restrictions on its density, which may not be readily available. A case in point is the proof that the null spaces of the integral operators for the interior Neumann and exterior Dirichlet problems are three-dimensional. The version in [2, Chapter VI, §3] is ultimately based on the assumption that if $V \varphi=0$ on the boundary, then $\varphi=0$, which is true in the three-dimensional theory but not always so in plane elasticity [3]. The new proof given in [4] in three dimensions makes use of the regularity of $V$ at infinity; as mentioned above, this property does not hold in the plane case. Even the proof indicated in $[5, \S 2.7]$ for the equations of bending of plates with transverse shear deformation cannot be adapted to plane strain, since, although it operates with the double-layer potential, which remains regular at infinity in two dimensions, it also relies essentially on the uniqueness of the solution of the exterior Neumann problem, unavailable under the conditions (1).

Other notable real variable angles of approach in plane elasticity can be found in [6], where the boundary value problems are solved in terms of biharmonic scalar functions, and in [7], where the integral equations are derived from the Somigliana formula. The equation for the Dirichlet problem in [7], however, is of the first kind and does not always have a unique solution [3]. A different formulation of this procedure has now been rigorously investigated in [8].

The aim of this paper is to give-for the first time, as far as the author is aware - a full and correct account of the real boundary integral equation method in application to plane strain. We propose a far-field pattern which guarantees the uniqueness of the solution of the exterior Neumann problem, indicate the physical significance of this pattern, and show how the solutions obtained by means of this technique generate those produced by the direct method.

The proofs are omitted where they follow the classical scheme, explicit mention being made only of those details that are specific to the case of plane elasticity.

In this form of presentation, the method can easily be adapted to other twodimensional elliptic systems in continuum mechanics.

\section{Preliminaries}

In what follows Greek and Latin subscripts take the values 1, 2 and 1, 2, 3, respectively, the convention of summation over repeated indices is understood, $\mathscr{M}_{m \times n}$ is the space of $(m \times n)$-matrices, $E_{n}$ is the identity element in $\mathscr{M}_{n \times n}$, a superscript $\mathrm{T}$ indicates matrix transposition, and $(\ldots),{ }_{\alpha}=\partial(\ldots) / \partial x_{\alpha}$. If $X$ is a space of scalar functions and $v$ a matrix, $v \in X$ means that every component of $v$ belongs to $X$. Also, if $\mathscr{L}$ is an operator defined on functions $\theta \in \mathscr{M}_{p \times 1}$ and such that $\mathscr{L} \theta \in \mathscr{M}_{r \times 1}$, and if $\Theta \in \mathscr{M}_{p \times q}$, then $\mathscr{L} \Theta \in \mathscr{M}_{r \times q}$ is the matrix with columns $(\mathscr{L} \boldsymbol{\Theta})^{(i)}=\mathscr{L}\left(\boldsymbol{\Theta}^{(i)}\right)$.

Let $S$ be a domain in $\mathbb{R}^{2}$ bounded by a closed $C^{2}$-curve $\partial S$ and occupied by a homogeneous and isotropic material with Lamé constants $\lambda$ and $\mu$. The state of plane strain is characterized by a displacement field $u=\left(u_{1}, u_{2}, u_{3}\right)^{\mathrm{T}}$ 
of the form

$$
u_{\alpha}=u_{\alpha}\left(x_{1}, x_{2}\right), \quad u_{3}=0,
$$

where $x=\left(x_{1}, x_{2}\right)$ is a generic point in $\mathbb{R}^{2}$. In the absence of body forces, (2) gives rise to the system of equilibrium equations

$$
A\left(\partial_{x}\right) u=0,
$$

in which now $u=\left(u_{1}, u_{2}\right)^{\mathrm{T}}, A\left(\partial_{x}\right)=A\left(\partial / \partial x_{1}, \partial / \partial x_{2}\right)$,

$$
A\left(\xi_{1}, \xi_{2}\right)=\left(\begin{array}{cc}
\mu \Delta+(\lambda+\mu) \xi_{1}^{2} & (\lambda+\mu) \xi_{1} \xi_{2} \\
(\lambda+\mu) \xi_{1} \xi_{2} & \mu \Delta+(\lambda+\mu) \xi_{2}^{2}
\end{array}\right),
$$

and $\Delta=\xi_{1}^{2}+\xi_{2}^{2}$.

We also consider the boundary stress operator $T\left(\partial_{x}\right)=T\left(\partial / \partial x_{1}, \partial / \partial x_{2}\right)$ defined by

$$
T\left(\xi_{1}, \xi_{2}\right)=\left(\begin{array}{cc}
(\lambda+2 \mu) \nu_{1} \xi_{1}+\mu \nu_{2} \xi_{2} & \mu \nu_{2} \xi_{1}+\lambda \nu_{1} \xi_{2} \\
\lambda \nu_{2} \xi_{1}+\mu \nu_{1} \xi_{2} & \mu \nu_{1} \xi_{1}+(\lambda+2 \mu) \nu_{2} \xi_{2}
\end{array}\right)
$$

where $\nu=\left(\nu_{1}, \nu_{2}\right)^{\mathrm{T}}$ is the unit outward normal to $\partial S$.

The internal energy density is given by

$$
E(u, u)=\frac{1}{2}\left[\lambda\left(u_{1,1}+u_{2,2}\right)^{2}+2 \mu\left(u_{1,1}^{2}+u_{2,2}^{2}\right)+\mu\left(u_{1,2}+u_{2,1}\right)^{2}\right] .
$$

We assume that

$$
\lambda+\mu>0, \quad \mu>0,
$$

in which case it is easy to see that the operator $A$ is elliptic and $E$ is a positive quadratic form. It can be shown that $E(u, u)=0$ if and only if

$$
u=\left(c_{1}+c_{0} x_{2}, c_{2}-c_{0} x_{1}\right)^{\mathrm{T}},
$$

where $c_{0}$ and $c_{\alpha}$ are arbitrary constants. This is the most general rigid displacement compatible with $(2)$. We take $\left\{F^{(i)}\right\}$ to be a basis for the space of such rigid displacements, where $F^{(i)}$ are the columns of the matrix

$$
F=\left(\begin{array}{rrr}
1 & 0 & x_{2} \\
0 & 1 & -x_{1}
\end{array}\right)
$$

Clearly, $A F=0$ in $\mathbb{R}^{2}, T F=0$ on $\partial S$, and a generic vector of the form (4) can be written as $F k$, where $k \in \mathscr{M}_{3 \times 1}$ is constant and arbitrary.

Let $S^{+}$be the bounded domain enclosed by $\partial S$ and $S^{-}=\mathbb{R}^{2} \backslash\left(S^{+} \cup \partial S\right)$. Direct verification shows that

$$
\int_{S^{+}} F^{\mathrm{T}} A u d \sigma=\int_{\partial S} F^{\mathrm{T}} T u d s .
$$

Also, the following assertion is proved without difficulty.

Theorem 1 (Betti formula). If $u \in C^{2}\left(S^{+}\right) \cap C^{1}\left(\bar{S}^{+}\right)$is a solution of (3) in $S^{+}$, then

$$
2 \int_{S^{+}} E(u, u) d \sigma=\int_{\partial S} u^{\mathrm{T}} T u d s
$$




\section{Fundamental solutions}

A Galerkin representation of the solution of (3) with the right-hand side replaced by $\delta(|x-y|) E_{2}$, where $\delta$ is the Dirac delta distribution, yields the matrix of fundamental solutions [9]

$$
D(x, y)=A^{*}\left(\partial_{x}\right) t(x, y),
$$

where $A^{*}$ is the adjoint of $A$ and $t$ satisfies

$$
\left(\operatorname{det} A\left(\partial_{x}\right)\right) t(x, y)=\mu(\lambda+2 \mu) \Delta^{2}(x) t(x, y)=-\delta(|x-y|),
$$

that is,

$$
t(x, y)=-[8 \pi \mu(\lambda+2 \mu)]^{-1}|x-y|^{2} \ln |x-y| .
$$

If $\left\{E_{\alpha \beta}\right\}$ is the standard ordered basis for the space of constant matrices in $\mathscr{M}_{2 \times 2}$, then

$D(x, y)=-\frac{1}{4 \pi \mu(\alpha+1)}\left[(2 \alpha \ln |x-y|+2 \alpha+1) E_{2}-2 \frac{\left(x_{\alpha}-y_{\alpha}\right)\left(x_{\beta}-y_{\beta}\right)}{|x-y|^{2}} E_{\alpha \beta}\right]$,

where $\alpha=(\lambda+3 \mu) /(\lambda+\mu)$. This shows that $D(x, y)=D^{\mathrm{T}}(x, y)=D(y, x)$. We also introduce the matrix of singular solutions of (3)

$$
P(x, y)=\left[T\left(\partial_{y}\right) D(y, x)\right]^{\mathrm{T}} .
$$

Explicitly, this is written as

$$
\begin{array}{r}
P(x, y)=-\frac{1}{2 \pi}\left\{\frac{\alpha-1}{\alpha+1} \varepsilon_{\alpha \beta}\left[\frac{\partial}{\partial s(y)} \ln |x-y|\right] E_{\alpha \beta}+\left[\frac{\partial}{\partial \nu(y)} \ln |x-y|\right] E_{2}\right. \\
\left.-\frac{2}{\alpha+1} \varepsilon_{\alpha \gamma}\left[\frac{\partial}{\partial s(y)} \frac{\left(x_{\alpha}-y_{\alpha}\right)\left(x_{\beta}-y_{\beta}\right)}{|x-y|^{2}}\right] E_{\gamma \beta}\right\},
\end{array}
$$

where $\varepsilon_{\alpha \beta}$ is the alternating tensor in the plane.

It is easily verified that $D^{(i)}(x, y)$ and $P^{(i)}(x, y)$ satisfy (3) at all $x \in \mathbb{R}^{2}$, $x \neq y$, and that

$$
D(x, y)=O(\ln |x|), \quad P(x, y)=O\left(|x|^{-1}\right) \quad \text { as } \quad|x| \rightarrow \infty, \quad y \in \partial S .
$$

\section{BoundaRY VALUE PROBLEMS AND UNIQUENESS OF SOLUTIONS}

We would like the Betti formula to hold in $S^{-}$for solutions that may include an arbitrary rigid displacement, but this cannot be achieved without restrictions on the behaviour of $u$ at infinity.

Consider the class $\mathscr{A}$ of vectors $u \in \mathscr{M}_{2 \times 1}$ whose components in terms of polar coordinates, as $r=|x| \rightarrow \infty$, are of the form

$$
\begin{aligned}
& u_{1}(r, \theta)=r^{-1}\left(\alpha m_{0} \sin \theta+m_{1} \cos \theta+m_{0} \sin 3 \theta+m_{2} \cos 3 \theta\right)+O\left(r^{-2}\right), \\
& u_{2}(r, \theta)=r^{-1}\left(m_{3} \sin \theta+\alpha m_{0} \cos \theta+m_{4} \sin 3 \theta-m_{0} \cos 3 \theta\right)+O\left(r^{-2}\right),
\end{aligned}
$$

where $m_{0}, \ldots, m_{4}$ are arbitrary constants. Also, let

$$
\mathscr{A}^{*}=\left\{u: u=F k+s^{\mathscr{A}}\right\},
$$

where $k \in \mathscr{M}_{3 \times 1}$ is constant and arbitrary and $s^{\mathscr{A}} \in \mathscr{M}_{2 \times 1} \cap \mathscr{A}$.

Let $\mathscr{P}, \mathscr{Q}, \mathscr{R}, \mathscr{S} \in \mathscr{M}_{2 \times 1}$ be prescribed on $\partial S$. We formulate the interior and exterior Dirichlet and Neumann problems as follows: 
$\left(\mathrm{D}^{+}\right)$Find $u \in C^{2}\left(S^{+}\right) \cap C^{1}\left(\bar{S}^{+}\right)$satisfying (3) in $S^{+}$and $\left.u\right|_{\partial S}=\mathscr{P}$.

$\left(\mathrm{N}^{+}\right)$Find $u \in C^{2}\left(S^{+}\right) \cap C^{1}\left(\bar{S}^{+}\right)$satisfying (3) in $S^{+}$and $\left.T u\right|_{\partial S}=\mathscr{Q}$.

$\left(\mathrm{D}^{-}\right)$Find $u \in C^{2}\left(S^{-}\right) \cap C^{1}\left(\bar{S}^{-}\right) \cap \mathscr{A}^{*}$ satisfying (3) in $S^{-}$and $\left.u\right|_{\partial S}=\mathscr{R}$.

$\left(\mathrm{N}^{-}\right)$Find $u \in C^{2}\left(S^{-}\right) \cap C^{1}\left(\bar{S}^{-}\right) \cap \mathscr{A}$ satisfying (3) in $S^{-}$and $\left.T u\right|_{\partial S}=\mathscr{S}$.

Theorem 2 (Betti formula). If $u \in C^{2}\left(S^{-}\right) \cap C^{1}\left(\bar{S}^{-}\right) \cap \mathscr{A}^{*}$ is a solution of (3) in $S^{-}$, then

$$
2 \int_{S^{-}} E(u, u) d \sigma=-\int_{\partial S} u^{\mathrm{T}} T u d s
$$

Proof. Let $\partial K_{R}$ be a circle with centre at $x$ and radius $R$ sufficiently large. Using (9), we see that on $\partial K_{R}$

$$
x_{2}(T u)_{1}-x_{1}(T u)_{2}=g(\theta) R^{-1}+O\left(R^{-2}\right), \quad \int_{0}^{2 \pi} g(\theta) d \theta=0
$$

hence, in view of $(8)$,

$$
\int_{\partial K_{R}} u^{\mathrm{T}} T u d s \rightarrow 0 \quad \text { as } \quad R \rightarrow \infty .
$$

The classical argument [2] can now be applied.

Theorem 3. (i) $\left(\mathrm{D}^{+}\right),\left(\mathrm{D}^{-}\right)$, and $\left(\mathrm{N}^{-}\right)$have at most one solution.

(ii) Any two solutions of $\left(\mathrm{N}^{+}\right)$differ by an arbitrary rigid displacement.

The assertion is proved by means of the well-known procedure that makes use of Theorems 1 and 2.

\section{Elastic potentials}

We introduce the single-layer potential

$$
(V \varphi)(x)=\int_{\partial S} D(x, y) \varphi(y) d s(y)
$$

and the double-layer potential

$$
(W \varphi)(x)=\int_{\partial S} P(x, y) \varphi(y) d s(y),
$$

where $\varphi \in \mathscr{M}_{2 \times 1}$. We also define a functional $p$ on continuous functions $\zeta \in \mathscr{M}_{2 \times 1}$ on $\partial S$ by

$$
p \zeta=\int_{\partial S} F^{\mathrm{T}} \zeta d s .
$$

Theorem 4. If $\varphi \in C(\partial S)$, then

(i) $W \varphi \in \mathscr{A}$;

(ii) $V \varphi \in \mathscr{A}$ if and only if $p \varphi=0$.

Proof. The first part of the assertion is obtained by direct verification. The second part follows from the fact that, as $r=|x| \rightarrow \infty$,

$$
(V \varphi)(r, \theta)=M^{\infty}(r, \theta) p \varphi+s^{\mathscr{A}}
$$


where

$$
\begin{aligned}
& 4 \pi \mu(\alpha+1) M^{\infty}(r, \theta) \\
& =\left(\begin{array}{ccr}
-2 \alpha(\ln r+1)+\cos 2 \theta & \sin 2 \theta & r^{-1}(\alpha+1) \sin \theta \\
\sin 2 \theta & -2 \alpha(\ln r+1)-\cos 2 \theta & -r^{-1}(\alpha+1) \cos \theta
\end{array}\right) .
\end{aligned}
$$

It can easily be verified that $A M^{\infty}=0$ in $\mathbb{R}^{2}$.

Theorem 5. (i) If $\varphi \in C(\partial S)$, then $V \varphi$ and $W \varphi$ are analytic and satisfy

$$
A(V \varphi)=A(W \varphi)=0 \text { in } S^{+} \cup S^{-} .
$$

(ii) If $\varphi \in C^{0, \alpha}(\partial S), \alpha \in(0,1)$, then the direct values $V_{0} \varphi$ and $W_{0} \varphi$ of $V \varphi$ and $W \varphi$ on $\partial S$ exist (the latter as principal value), the functions

$$
\mathscr{V}^{+}(\varphi)=\left.(V \varphi)\right|_{S^{+}}, \quad \mathscr{V}^{-}(\varphi)=\left.(V \varphi)\right|_{S^{-}}
$$

are of class $C^{\infty}\left(S^{+}\right) \cap C^{1, \alpha}\left(\bar{S}^{+}\right)$and $C^{\infty}\left(S^{-}\right) \cap C^{1, \alpha}\left(\bar{S}^{-}\right)$, respectively, and

$$
T \mathscr{V}^{+}(\varphi)=\left(W_{0}^{*}+\frac{1}{2} I\right) \varphi, \quad T \mathscr{V}^{-}(\varphi)=\left(W_{0}^{*}-\frac{1}{2} I\right) \varphi,
$$

where $W_{0}^{*}$ is the adjoint of $W_{0}$ and $I$ the identity operator.

(iii) If $\varphi \in C^{1, \alpha}(\partial S), \alpha \in(0,1)$, then the functions

$$
\mathscr{W}^{+}(\varphi)=\left\{\begin{array}{ll}
\left.(W \varphi)\right|_{S^{+}} & \text {in } S^{+}, \\
\left(W_{0}-\frac{1}{2} I\right) \varphi & \text { on } \partial S,
\end{array} \quad \mathscr{W}^{-}(\varphi)= \begin{cases}\left.(W \varphi)\right|_{S^{-}} & \text {in } S^{-}, \\
\left(W_{0}+\frac{1}{2} I\right) \varphi & \text { on } \partial S\end{cases}\right.
$$

are of class $C^{\infty}\left(S^{+}\right) \cap C^{1, \alpha}\left(\bar{S}^{+}\right)$and $C^{\infty}\left(S^{-}\right) \cap C^{1, \alpha}\left(\bar{S}^{-}\right)$, respectively, and $T \mathscr{W}^{+}(\varphi)=T \mathscr{W}^{-}(\varphi)$ on $\partial S$.

Proof. Part (i) follows from the classical argument for systems of partial differential equations [10] and direct verification.

For parts (ii) and (iii), we use (6) and (7) to find that

$$
\begin{aligned}
& V \varphi=\frac{1}{4 \pi \mu(\alpha+1)}\left[2 \alpha v \varphi+(2 \alpha+1) \mathcal{I} \varphi-2 E_{\alpha \beta}\left(v_{\alpha \beta}^{b} \varphi\right)\right], \\
& W \varphi=\frac{1}{2 \pi}\left[-\frac{\alpha-1}{\alpha+1} \varepsilon_{\alpha \beta} E_{\alpha \beta}\left(v^{f} \varphi\right)+w \varphi+\frac{2}{\alpha+1} \varepsilon_{\alpha \gamma} E_{\gamma \beta}\left(v_{\alpha \beta}^{e} \varphi\right)\right],
\end{aligned}
$$

where

$$
\begin{aligned}
& (v \varphi)(x)=-\int_{\partial S}(\ln |x-y|) \varphi(y) d s(y) \\
& (w \varphi)(x)=-\int_{\partial S}\left[\frac{\partial}{\partial \nu(y)} \ln |x-y|\right] \varphi(y) d s(y), \\
& \left(v_{\alpha \beta}^{b} \varphi\right)(x)=\int_{\partial S} \frac{\left(x_{\alpha}-y_{\alpha}\right)\left(x_{\beta}-y_{\beta}\right)}{|x-y|^{2}} \varphi(y) d s(y), \\
& \left(v_{\alpha \beta}^{e} \varphi\right)(x)=\int_{\partial S}\left[\frac{\partial}{\partial s(y)} \frac{\left(x_{\alpha}-y_{\alpha}\right)\left(x_{\beta}-y_{\beta}\right)}{|x-y|^{2}}\right] \varphi(y) d s(y), \\
& \left(v^{f} \varphi\right)(x)=\int_{\partial S}\left[\frac{\partial}{\partial s(y)} \ln |x-y|\right] \varphi(y) d s(y),
\end{aligned}
$$


and

$$
\mathscr{J} \varphi=\int_{\partial S} \varphi d s
$$

The result now follows from the behaviour of all these functions in the neighbourhood of $\partial S$, which has been investigated in detail in $[5, \S \S 1.5,1.6]$.

Theorem 6 (Somigliana formulae). (i) If $u \in C^{2}\left(S^{+}\right) \cap C^{1}\left(\bar{S}^{+}\right)$is a solution of (3) in $S^{+}$, then

$$
\int_{\partial S}\left[D(x, y) T\left(\partial_{y}\right) u(y)-P(x, y) u(y)\right] d s(y)= \begin{cases}u(x), & x \in S^{+}, \\ \frac{1}{2} u(x), & x \in \partial S, \\ 0, & x \in S^{-} .\end{cases}
$$

(ii) If $u \in C^{2}\left(S^{-}\right) \cap C^{1}\left(\bar{S}^{-}\right)$is a solution of (3) in $S^{-}$and $u=O\left(|x|^{-1}\right)$, $u,_{\alpha}=O\left(|x|^{-2}\right)$ as $|x| \rightarrow \infty$, then

$$
-\int_{\partial S}\left[D(x, y) T\left(\partial_{y}\right) u(y)-P(x, y) u(y)\right] d s(y)= \begin{cases}0, & x \in S^{+} \\ \frac{1}{2} u(x), & x \in \partial S \\ u(x), & x \in S^{-}\end{cases}
$$

The first part of this assertion is proved in the usual way [2]. The second part, however, appears to be addressed incorrectly in [2, p. 187], where it is claimed that it holds in this form if $u=O(1), u,_{\alpha}=O\left(|x|^{-2}\right)$ as $|x| \rightarrow \infty$. The asymptotic relations $(8)$ show that this is not the case, and that the conditions in Theorem 6(ii) are sufficient for the result to hold.

\section{EXISTENCE OF SOLUTIONS}

If we seek the solutions of $\left(\mathrm{D}^{+}\right),\left(\mathrm{D}^{-}\right),\left(\mathrm{N}^{+}\right)$, and $\left(\mathrm{N}^{-}\right)$in the form of $\mathscr{W}^{+}(\varphi), \mathscr{W}^{-}(\varphi)+F k, \mathscr{V}^{+}(\varphi)$, and $\mathscr{V}^{-}(\varphi)$, respectively, then, by Theorem 5 , these problems reduce to the boundary integral equations

$\begin{array}{lc}\left(\mathscr{D}^{+}\right) & \left(W_{0}-\frac{1}{2} I\right) \varphi=\mathscr{P}, \\ \left(\mathscr{N}^{+}\right) & \left(W_{0}^{*}+\frac{1}{2} I\right) \varphi=\mathscr{Q}, \\ \left(\mathscr{D}^{-}\right) & \left(W_{0}+\frac{1}{2} I\right) \varphi=\mathscr{R}-F k, \\ \left(\mathscr{N}^{-}\right) & \left(W_{0}^{*}-\frac{1}{2} I\right) \varphi=\mathscr{S} .\end{array}$

It is clear from the second formula (11) that these equations are singular.

Theorem 7. (i) $\frac{1}{2}$ is not an eigenvalue of $W_{0}$ (hence, nor of $W_{0}^{*}$ ).

(ii) $-\frac{1}{2}$ is an eigenvalue of $W_{0}$ and of $W_{0}^{*}$. The null spaces of $W_{0}+\frac{1}{2} I$ and of $W_{0}^{*}+\frac{1}{2} I$ are three-dimensional subspaces of $C^{1, \alpha}(\partial S)$. The former is spanned by $\left\{F^{(i)}\right\}$; the latter is spanned by a set of three linearly independent vectors $\left\{\Phi^{(i)}\right\}$ which may be chosen uniquely so that $F$ and the matrix $\Phi \in \mathscr{M}_{2 \times 3}$ are biorthonormal, that is,

$$
p \Phi=E_{3} .
$$

This is shown exactly as in [5, Theorem 2.40], since, by Theorems 3 and 4, $W \varphi$ has the required behaviour at infinity and $\left(\mathrm{N}^{-}\right)$has at most one solution. The last part of (ii) was proved in [3]. 
Setting, in turn, $u=F^{(i)}$ in Theorem 6(i) for $x \in \partial S$, we see that

$$
\int_{\partial S} P(x, y) d s(y)=-\frac{1}{2} E_{2}, \quad \int_{\partial S} \varepsilon_{\alpha \beta} y_{\beta} P_{\gamma \alpha}(x, y) d s(y)=-\frac{1}{2} \varepsilon_{\gamma \beta} x_{\beta} .
$$

If we now integrate $\left(\mathscr{N}^{-}\right)$and $\varepsilon_{\alpha \beta} x_{\beta}\left(\mathscr{N}^{-}\right)_{\alpha}$ over $\partial S$ and use the above equalities, we obtain

$$
p \varphi=-p \mathscr{S} \text {. }
$$

Theorem 8. (i) $\left(\mathrm{D}^{+}\right)$has a unique solution for any $\mathscr{P} \in C^{1, \alpha}(\partial S)$, which can be represented as $\mathscr{W}^{+}(\varphi)$ with $\varphi \in C^{1, \alpha}(\partial S)$.

(ii) $\left(\mathrm{N}^{-}\right)$has a unique solution for any $\mathscr{S} \in C^{0, \alpha}(\partial S)$ if and only if $p \mathscr{S}=$ 0 . The solution can be represented as $\mathscr{V}^{-}(\varphi)$ with $\varphi \in C^{0, \alpha}(\partial S)$.

(iii) $\left(\mathrm{N}^{+}\right)$is soluble for any $\mathscr{Q} \in C^{0, \alpha}(\partial S)$ if and only if $p \mathscr{Q}=0$. The solution is unique up to a matrix of the form $F k$, where $k \in \mathscr{M}_{3 \times 1}$ is constant and arbitrary, and can be represented as $\mathscr{V}^{+}(\varphi)$ with $\varphi \in C^{0, \alpha}(\partial S)$.

(iv) $\left(\mathrm{D}^{-}\right)$has a unique solution for any $\mathscr{R} \in C^{1, \alpha}(\partial S)$, which can be represented as the sum of $\mathscr{W}^{-}(\varphi)$ with $\varphi \in C^{1, \alpha}(\partial S)$ and a specific matrix $F k$.

The assertion is proved in the usual way (see, for example, [5, $\$ 2.7]$ ), by means of Theorems 3-7 and the Fredholm Alternative; the latter is applicable since, as can readily be checked, the index [11] of the singular integral equations involved is equal to zero. In view of (12), the solvability condition for $\left(\mathscr{D}^{-}\right)$ is satisfied if we choose

$$
k=\int_{\partial S} \Phi^{\mathrm{T}} \mathscr{R} d s .
$$

\section{CONNECTION WITH THE DIRECT METHOD}

In this section we show how the solutions of the various equations produced by the direct method (based on the Somigliana relations) can be expressed in terms of the solutions constructed by means of the above technique.

The boundary integral operators introduced in conjunction with the elastic potentials satisfy the composition relations [8]

$$
\begin{array}{llll}
W_{0} V_{0}=V_{0} W_{0}^{*}, & N_{0} V_{0}=W_{0}^{* 2}-\frac{1}{4} I & \text { on } & C^{0, \alpha}(\partial S), \\
N_{0} W_{0}=W_{0}^{*} N_{0}, & V_{0} N_{0}=W_{0}^{2}-\frac{1}{4} I & \text { on } & C^{1, \alpha}(\partial S),
\end{array}
$$

where $N_{0}$ is the operator defined on $C^{1, \alpha}(\partial S)$ by

$$
N_{0} f=T \mathscr{W}^{+}(f)=T \mathscr{W}^{-}(f) .
$$

The Neumann problems. We rewrite the Somigliana relation for a solution of (3) in $S^{+}$(Theorem 6(i)) as

$$
\mathscr{V}^{+}\left(\left.T u\right|_{\partial S}\right)-\mathscr{W}^{+}\left(\left.u\right|_{\partial S}\right)=u \text { in } \bar{S}^{+} .
$$

For $\left(\mathrm{N}^{+}\right)$, let $\left.T u\right|_{\partial S}=\mathscr{Q}$ (known) and $\left.u\right|_{\partial S}=\psi$ (unknown). Then (17) yields $\left(\bar{N}^{+}\right)$

$$
\left(W_{0}+\frac{1}{2} I\right) \psi=V_{0} \mathscr{Q} .
$$

Applying $V_{0}$ to $\left(\mathscr{N}^{+}\right)$and using (15), we see that

$$
V_{0}\left(W_{0}^{*}+\frac{1}{2} I\right) \varphi=\left(W_{0}+\frac{1}{2} I\right)\left(V_{0} \varphi\right)=V_{0} \mathscr{Q},
$$


which, subtracted from $\left(\overline{\mathscr{N}}^{+}\right)$, yields $\left(W_{0}+\frac{1}{2} I\right)\left(\psi-V_{0} \varphi\right)=0$. By Theorem 7(ii), this means that

$$
\psi=V_{0} \varphi+F k,
$$

where $k \in \mathscr{M}_{3 \times 1}$ is constant and arbitrary. The arbitrariness in (18) is justified, since the solutions of both $\left(\mathscr{N}^{+}\right)$and $\left(\overline{\mathscr{N}}^{+}\right)$are not unique.

For $\left(\mathrm{N}^{-}\right)$, we need $u \in \mathscr{A}$. Then Theorem 6(ii) holds, and we rewrite the corresponding Somigliana formula as

$$
-\mathscr{V}^{-}\left(\left.T u\right|_{\partial S}\right)+\mathscr{W}^{-}\left(\left.u\right|_{\partial S}\right)=u \text { in } \bar{S}^{-} .
$$

Once more, let $\left.T u\right|_{\partial S}=\mathscr{S}$ (known) and $\left.u\right|_{\partial S}=\psi$ (unknown). From (19) we see that

$$
\left(W_{0}-\frac{1}{2} I\right) \psi=V_{0} \mathscr{S} .
$$

Proceeding as above but operating with $\left(\mathscr{N}^{-}\right)$, we find that $\left(W_{0}-\frac{1}{2} I\right) \times$ $\left(\psi-N_{0} \varphi\right)=0$, from which

$$
\psi=V_{0} \varphi,
$$

since, by Theorem 7(ii), $\frac{1}{2}$ is not an eigenvalue of $W_{0}$. The solvability condition $p \mathscr{S}=0$ for the exterior Neumann problem, (13), and Theorem 4 ensure that $u$ indeed belongs to $\mathscr{A}$, as required.

The Dirichlet problems. These can be approached within the framework of the direct method in two ways.

First version. For $\left(\mathrm{D}^{+}\right)$, let $\left.u\right|_{\partial S}=\mathscr{P}$ (known) and $\left.T u\right|_{\partial S}=\psi$ (unknown). If $(17)$ is used again, we obtain

$$
V_{0} \psi=\left(W_{0}+\frac{1}{2} I\right) \mathscr{P},
$$

which is an equation of the first kind.

In [3] it is shown that for every $C^{2}$-boundary curve $\partial S$ there exists a unique constant $\mathscr{C} \in \mathscr{M}_{3 \times 3}$ such that $V_{0} \Phi=F \mathscr{C}$, and that if $\mathscr{C}$ is singular, then $\left(\bar{D}^{+}\right)$does not have a unique solution. More specifically, the null space of $V_{0}$ consists of all $f=\Phi a$, where $a \in \mathscr{M}_{3 \times 1}$ is any constant vector such that $\mathscr{C} a=0$.

Since $\psi=\left.T u\right|_{\partial S}$, we see that, by (5), $\psi$ must also satisfy

$$
p \psi=\int_{\partial S} F^{\mathrm{T}} T u d s=\int_{S^{+}} F^{\mathrm{T}} A u d \sigma=0 .
$$

If $\operatorname{det} \mathscr{C} \neq 0$, then $\left(\mathscr{D}^{+}\right)$has a unique solution. Applying $W_{0}+\frac{1}{2} I$ to $\left(\mathscr{D}^{+}\right)$ and using (15), we obtain

$$
\left(W_{0}^{2}-\frac{1}{4} I\right) \varphi=V_{0} N_{0} \varphi=\left(W_{0}+\frac{1}{2} I\right) \mathscr{P},
$$

which, subtracted from $\left(\overline{\mathscr{D}}^{+}\right)$, leads to $V_{0}\left(\psi-N_{0} \varphi\right)=0$; hence, we conclude that

$$
\psi=N_{0} \varphi .
$$

This also shows that $\psi$ satisfies $(20)$, since $p N_{0}=0$ on $C^{1, \alpha}(\partial S)$ [8].

If $\operatorname{det} \mathscr{C}=0$, then $\psi=N_{0} \varphi+\Phi a$ for any $a$ such that $\mathscr{C} a=0$. But this arbitrariness is spurious, because to satisfy (20), in view of (12) we must have $a=0$, so we end up once more with the formula (21). 
For $\left(\mathrm{D}^{-}\right)$, let $\left.u\right|_{\partial S}=\mathscr{R}$ (known) and $\left.T u\right|_{\partial S}=\psi$ (unknown). Here the solution is sought in $\mathscr{A}^{*}$, that is, $u=u^{\mathscr{A}}+F k$. (Because of the formulation of the problem in terms of the class $\mathscr{A}^{*}$ and the uniqueness of its solution, it is obvious that the rigid displacement is the same as that corresponding to (14) in Theorem 8(iv).) Consequently, (19) holds for $u-F k \in \mathscr{A}$. Since $\left.(u-F k)\right|_{\partial S}=\mathscr{R}-F k$ and $\left.T(u-F k)\right|_{\partial S}=\left.T u\right|_{\partial S}=\psi,(19)$ yields the integral equation of the first kind

$$
V_{0} \psi=\left(W_{0}-\frac{1}{2} I\right)(\mathscr{R}-F k) .
$$

Also, from (19) it follows that

$$
-V \psi+W(\mathscr{R}-F k)=u-F k \text { in } S^{-} .
$$

Since the last two terms in (22) belong to $\mathscr{A}$, we deduce that so does $V \psi$. Consequently, by Theorem 4(ii), $\psi$ must again satisfy the condition $p \psi=0$. (This can also be verified by direct calculation.) An argument similar to that in the case of $\left(\mathrm{D}^{+}\right)$now leads to $(21)$.

Using the solution (21) of $\left(\mathrm{D}^{-}\right)$, we can also solve a more general exterior Dirichlet problem, which requires to find $v \in C^{2}\left(S^{-}\right) \cap C^{1}\left(\bar{S}^{-}\right)$such that

$$
\begin{gathered}
A v=0 \text { in } S^{-}, \\
\left.v\right|_{\partial S}=\mathscr{R}, \\
v=M^{\infty} q+v^{\mathscr{A}^{*}} \text { as }|x| \rightarrow \infty,
\end{gathered}
$$

where $q \in \mathscr{M}_{3 \times 1}$ is a prescribed constant vector. At this stage we already know from (22) that $u=-\mathscr{V}^{-}(\psi)+\mathscr{W}^{-}(\mathscr{R}-F k)+F k$ satisfies the first two equalities (23). However, $u=u^{\mathscr{A}^{*}}$ as $|x| \rightarrow \infty$, which is not good enough if $q \neq 0$. To get the right result, we consider a solution for (23) of the form

$$
v=u+\mathscr{V}^{-}(\Phi q)-F l,
$$

where $l \in \mathscr{M}_{3 \times 1}$ is a constant vector to be determined. Taking (10) and (12) into account, we see immediately that $v$ satisfies the first and third relations (23) for any $l$, and the second one if $l=\mathscr{C} q$. Thus, in view of (21), the (unique) solution of the problem (23) is

$$
v=\mathscr{V}^{-}\left(\Phi q-N_{0} \varphi\right)+\mathscr{W}^{-}(\mathscr{R})+F(k-\mathscr{C} q),
$$

since, as can easily be checked, $\mathscr{W}^{-}(F)=0$.

Second version. A modified approach in the direct method enables us to avoid the use of equations of the first kind for the Dirichlet problems. Thus, if we apply $T$ to the Somigliana formulae (17) and (19) (with $u$ replaced by $u-F k$ ), then, by Theorem $5,(16)$, and the fact that $\mathscr{W}^{-}(F)=0$, we arrive at the equations

$$
\begin{array}{ll}
\left(\tilde{\mathscr{D}}^{+}\right) & \left(W_{0}^{*}-\frac{1}{2} I\right) \psi=N_{0} \mathscr{P}, \\
\left(\tilde{\mathscr{D}}^{-}\right) & \left(W_{0}^{*}+\frac{1}{2} I\right) \psi=N_{0} \mathscr{R} .
\end{array}
$$

Taking Theorem 7(ii) into account, we deduce that $\left(\tilde{\mathscr{D}}^{+}\right)$has a unique solution, and that $\left(\tilde{\mathscr{D}}^{-}\right)$is solvable since

$$
\int_{\partial S} F^{\mathrm{T}} N_{0} \mathscr{R} d s=p N_{0} \mathscr{R}=0 .
$$


The solution of ( $\tilde{\mathscr{D}}^{-}$) is unique up to a term of the form $\Phi a$, with $a \in \mathscr{M}_{3 \times 1}$ constant and arbitrary.

Applying $N_{0}$ to $\left(\mathscr{D}^{+}\right)$and using (15), we find that

$$
N_{0}\left(W_{0}-\frac{1}{2} I\right) \varphi=\left(W_{0}^{*}-\frac{1}{2} I\right)\left(N_{0} \varphi\right)=N_{0} \mathscr{P} .
$$

We now eliminate $N_{0} \mathscr{P}$ between this equality and $\left(\tilde{\mathscr{D}}^{+}\right)$to obtain

$$
\left(W_{0}^{*}-\frac{1}{2} I\right)\left(\psi-N_{0} \varphi\right)=0 .
$$

Since $\frac{1}{2}$ is not an eigenvalue of $W_{0}^{*}$, this leads back to $(21)$.

A similar argument in the case of $\left(\mathscr{D}^{-}\right)$and $\left(\tilde{\mathscr{D}}^{-}\right)$brings us to

$$
\left(W_{0}^{*}+\frac{1}{2} I\right)\left(\psi-N_{0} \varphi\right)=0,
$$

from which $\psi=N_{0} \varphi+\Phi a$. But $p \psi=0$ (here $\psi=\left.T u\right|_{\partial S}$, as in the first version of the method), so we conclude once more that $\varphi$ and $\psi$ satisfy (21).

\section{ConClusions}

The real boundary integral equation method developed in $\S \S 4-6$ represents the extension to plane elasticity of the corresponding three-dimensional technique [4]. Here, however, the need arises to restrict the solutions of the exterior problems to the finite energy classes $\mathscr{A}$ and $\mathscr{A}^{*}$. Far from being an artificial mathematical requirement, these classes have an acceptable physical meaning.

The general analytic solution of (3) in $S^{-}$is [1, Chapter V]

$$
2 \mu\left(u_{1}+i u_{2}\right)=\alpha \Omega(z)-z \overline{\Omega^{\prime}(z)}-\overline{\omega(z)} .
$$

If $p\left(\left.T u\right|_{\partial S}\right)=0$ and the stresses and rotation at infinity are zero, then the complex potentials above are

$$
\Omega(z)=a z^{-1}+O\left(|z|^{-2}\right), \quad \omega(z)=b z^{-1}+O\left(|z|^{-2}\right), \quad a \in \mathbb{C}, \quad b \in \mathbb{R},
$$

and (24) yields the far-field pattern (9), with

$$
m_{0}=\operatorname{Im} a, \quad m_{1}=\alpha \operatorname{Re} a-b, \quad m_{3}=-(\alpha \operatorname{Re} a+b), \quad m_{2}=m_{4}=\operatorname{Re} a .
$$

This implies that a solution of class $\mathscr{A}$ corresponds to a plane problem where the stresses and rotation vanish at infinity.

To guarantee that the solution belongs to $\mathscr{A}$, for $\left(\mathrm{N}^{-}\right)$we must require that the total stress acting on $\partial S$ be zero (Theorem $8(\mathrm{ii})$ ), which is not necessary in the three-dimensional theory.

As shown in $\S 7$, the above method seems to underpin the results of the direct method in the sense that the solutions of the latter can be expressed in terms of those of the former by means of operators intrinsically connected with the mathematical structure of the argument.

\section{REFERENCES}

1. N.I. Muskhelishvili, Some basic problems in the mathematical theory of elasticity, 3rd ed., Noordhoff, Groningen, 1949.

2. V.D. Kupradze, Potential methods in the theory of elasticity, Israel Program for Scientific Translations, Jerusalem, 1965.

3. C. Constanda, On non-unique solutions of weakly singular integral equations in plane elasticity, Quart. J. Mech. Appl. Math. 47 (1994), 261-267. 
4. V.D. Kupradze et al., Three-dimensional problems of the mathematical theory of elasticity and thermoelasticity, North-Holland, Amsterdam, 1979.

5. C. Constanda, A mathematical analysis of bending of plates with transverse shear deformation, Longman, Harlow, 1990.

6. M.A. Jaswon and G.T. Symm, Integral equation methods in potential theory and elastostatics, Academic Press, London, New York, and San Francisco, 1977.

7. F.J. Rizzo, An integral equation approach to boundary value problems of classical elastostatics, Quart. Appl. Math. 25 (1967), 83-95.

8. C. Constanda, Integral equations of the first kind in plane elasticity, Quart. Appl. Math. (to appear).

9. __ Some comments on the integration of certain systems of partial differential equations in continuum mechanics, J. Appl. Math. Phys. 29 (1978), 835-839.

10. C. Miranda, Partial differential equations of elliptic type, 2nd ed., Springer-Verlag, Berlin, 1970.

11. N.I. Muskhelishvili, Singular integral equations, Noordhoff, Groningen, 1946.

Department of Mathematics, University of Strathclyde, Glasgow, Scotland, United KINGDOM

E-mail address: C.Constandaestrath.ac.uk 